\title{
Patterns of sexual risk behavior among men who have sex with men in major cities of Myanmar: A cross- sectional study
}

\author{
Kyaw Min Htut ${ }^{\text {*\#, }}$, Chitlada Areesantichai ${ }^{\circledR}$, Myo Myo Mon*, Phyo Aung Naing* \\ * Department of Medical Research, Ministry of Health and Sports, Yangon, Myanmar \\ \#College of Public Health Sciences, Chulalongkorn University, Bangkok, Thailand \\ ${ }^{\circledR}$ Drug Dependence Research Centre, WHO Collaborating Centre for Research and Training in Drug Dependence, Health and Social Science and \\ Addition Research Unit, College of Public Health Sciences, Chulalongkorn University, Bangkok, Thailand \\ DOI: 10.29322/IJSRP.10.10.2020.p10653 \\ http://dx.doi.org/10.29322/IJSRP.10.10.2020.p10653
}

\begin{abstract}
Introduction: Globally, transmissions of diseases are more likely to be infected among MSM due to their sexual risk behaviors like unprotected anal sex and multiple sexual partners. Very scarce or limited studies were noted regarding alcohol consumption and sexual risk behavior among MSM in Myanmar.
\end{abstract}

Methods: A cross-sectional study was conducted to identify the patterns sexual risk behavior among MSM in two major cities of Myanmar. Purposive sampling was applied and sampling was made through Myanmar MSM network. Face-to-face interviews were conducted by using a structured questionnaire. Patterns of sexual risk behavior were described as frequency/percentage and mean/median as appropriate. Bivariate analysis (Chi-squared test) was also done to find out the association between condomless anal intercourse and multiple sexual partners.

Results: A total of 256 MSM included in the study and their mean age was 27.33 \pm 7.7 years. The age group was categorized into 1524 years as young MSM and $\geq 25$ years as adult MSM. All MSM in the study have experienced sexual intercourse with men in their lifetime. Within one month, $47.3 \%$ of young MSM and 63.9\% of adult MSM had multiple sexual partners. Over 70\% to $80 \%$ of MSM had multiple sexual partners within six months and one year respectively. Never/inconsistent condom use was detected among $61.3 \%$ of young MSM and 56.1\% of adult MSM who had sex with their permanent sexual partners within one month. On the other hand, $33.3 \%$ of young MSM and 23.5\% of adult MSM did not use condom regularly during one month. Significantly higher proportion of Apwint (open type) of MSM had more than one sexual partner than other types of MSM ( $\mathrm{p}<0.001$ ). Over 69\% of Apwint (open type) of MSM, 51.3\% of Apone (hidden type) and 26.5\% of Thange (partner of MSM) had more than one sexual partner. Similar pattern of association between type of MSM and number of partners was also seen for other time periods like within three months, six months and one year $(\mathrm{p}<0.001)$.

Conclusion: Current study identified the alcohol consumption and sexual risk behavior among MSM in major cities of Myanmar. Intervention to reduce unsafe sexual practices like having multiple partners and condomless anal intercourse should be done since many MSM had practiced these unsafe sexual behaviors.

Index Terms- Sexual risk behavior, Men who have sex with men, Myanmar

\section{INTRODUCTION}

Globally, transmissions of diseases are more likely to be infected among MSM due to their sexual risk behaviors like unprotected anal sex and multiple sexual partners. According to the previous studies, 40-70\% of MSM had sex without using condom (Herrera et al.; Li 
et al.). The prevalence of condomless anal intercourse (CAI) and their associated factors among MSM in China was documented in previous studies in China. Prevalence of CAI with male partners in the past six months was $43.7 \%$ and condomless vaginal intercourse was 21.6\%. CAI was associated with earlier homosexual debut, suicidal inclinations, childhood sexual abuse and HIV testing in recent year ( $\mathrm{Li}$ et al.). In a study conducted in Peru, condomless anal intercourse was associated with having multiple sexual partners. Similarly, having multiple sexual partners was very common and it may lead the risk of sexually transmitted infections. Previous studies documented that six to ten sexual partners were found among MSM within previous year. In addition, 50-70\% of MSM had multiple sexual partners. One of the studies stated that, there was sexual relationship with ten sexual partners within last one year (Chittamuru et al.; Garcia et al.; Pines, Karris and Little).

Sexual risk behaviours among MSM have been documented in previous studies. According to a systematic review of studies done in high-income countries, increasing trend of sexual risk behavior was seen among MSM such as condomless anal sex, condomless anal sex with casual partners and main partners, condomless anal sex with partners of unknown or discordant HIV status. Similarly, sexual risk behaviors among MSM were reported in many Asian countries. A study conducted in Malaysia identified that having multiple sexual partners was common and about $60 \%$ of them had unprotected sex within six months (Koh et al.). Likewise, in Vietnam, $70 \%$ among 1695 MSM had multiple sexual multiple sexual partnerships in the last 6 months. More than half of them also reported penetrative sex with concurrent partners within last 6 months (Garcia et al.). All these sexual risk behavior were crucial for further HIV transmission among MSM population.

In Myanmar, according to "Integrated Biological and Behavioral Surveillance Survey" (IBBS) data, median age at first anal sex with male was found at 17 years of age. In addition, proportion of MSM respondents those who had first sexual partner with male was documented as 95\% in Yangon and 84\% in Mandalay. Then, the proportion of MSM who had anal sex with men within one year was detected as 20\% in Yangon and 14\% in Mandalay. IBBS conducted in 2015 found that experience of being forced to have sex sometimes in the last 12 months ranged widely from 7\% to 40\% among the MSM in five study sites (National_AIDS_Program).

According to the local terminology in Myanmar, there were three common subgroups of MSM such as Apwint or open type, Apone or hidden type and Tha Nge. Apwint or open MSM are defined as "individuals born biological male but who openly express themselves femininely by dress and/or social interactions". Apone or hidden MSM are defined as "individuals born biological males who may also want to express themselves femininely but may not disclose this behavior to all segments of their social networks". Tha Nge are defined as "having a masculine outward appearance but have sex with men and insertive type in nature" (National_AIDS_Program).

In Myanmar, HIV is concentrated among key affected population like MSM whereas HIV prevalence was over $10 \%$ among them (UNAIDS). Very little or no known study was reported sexual risk behavior among MSM. Therefore, current study was conducted to identify sexual risk behavior in terms of condomless anal intercourse and multiple sexual partners (more than one sexual partner) among MSM.

\section{METHODS}

\section{Study design, population and area}

A cross-sectional study was conducted among MSM those aged more than 18 years in Yangon and Mandalay, major cities of Myanmar.

Inclusion criteria

- $\quad$ The one who identify himself as a man who have sex with man

- $\quad$ MSM who had engaged insertive or receptive anal sex or both

This publication is licensed under Creative Commons Attribution CC BY. 


\section{Sampling and sample size}

Purposive sampling was applied and MSMs were recruited through Myanmar MSM network. By considering the estimated proportion of MSM who have sex with men as $20 \%$, $95 \%$ confidence level and an error of $5 \%$, minimum required sample size was 246 (National_AIDS_Program).

\section{Data collection}

One day training of the interviewers was done at Department of Medical Research and pre-test was done at a non-study township in Yangon Region. Eligible participants were invited to participate in the study and recruited at the venues where MSMs usually gather like drop-in-centers of International Non-governmental Organizations, beauty parlours and others included the hotspot places of MSM where they usually gather such as home of famous beautician, moat and office of community based organization. After getting the informed consent, data collection was done by face-to-face interview. Strict adherence to ethical principles were ensured throughout the data collection period in order to maintain the confidentiality of the information of the study participants.

\section{Data management and analysis}

Data entry was carried out with EpiData version 3.1 and data analysis was done with SPSS version 21. Exploratory data analysis was done to check the errors, consistencies and missing values. Descriptive information regarding sexual risk behaviors was shown as frequency/percentage according to the age group of MSM. Bivariate analysis using chi-square statistic was also done to identify the association between number of sexual partners and types of MSM.

\section{Ethical considerations}

The proposal was submitted to Institutional Review Board, Department of Medical Research, Myanmar. Informed consents were taken from the participants after thorough explanation about the objectives of the study. Confidentiality and anonymity of the information were strictly ensured. All answer sheets and data reports were kept in locked cabinet.

\section{Operational definitions}

According to the local terminology in Myanmar, three groups of MSM were included in the study: Apwint or open type, Apone or hidden type and Tha Nge. Apwint or open MSM are defined as individuals born biological male but who openly express themselves femininely by dress and/or social interactions. Apone or hidden MSM are defined as individuals born biological males who may also want to express themselves femininely but may not disclose this behaviour to all segments of their social networks. Tha Nge are defined as having a masculine outward appearance but have sex with men" (National_AIDS_Program). Multiple sexual partners can be defined as having sex with two or more sexual partners.

\section{FINDINGS}

A total of $256 \mathrm{MSM}$ included in the study and mean age of MSM was 27.33 \pm 7.7 years. Tables were presented according to the age group that was categorized as $15-24$ years as young MSM and $\geq 25$ years as adult MSM. As shown in Table (1), 39\% of young MSM and $59 \%$ of adult MSM were "apwint" (open type).

Table 1 Socio-demographic characteristics of MSM from Yangon and Mandalay $(\mathrm{n}=256)$ 


\begin{tabular}{|c|c|c|}
\hline \multirow[t]{3}{*}{ Characteristics } & \multicolumn{2}{|c|}{ Age group (n=256) } \\
\hline & 15-24 years & $\geq 25$ years \\
\hline & $\mathbf{n}(\%)$ & $\mathrm{n}(\%)$ \\
\hline \multicolumn{3}{|l|}{ Type of MSM } \\
\hline Apwint (open) & $43(39.1)$ & $86(58.9)$ \\
\hline Apone (hidden) & $27(24.5)$ & $41(28.1)$ \\
\hline Thange (partner of apwint/apone) & $40(36.4)$ & $19(13.0)$ \\
\hline \multicolumn{3}{|l|}{ Income earning job } \\
\hline No/ not regular & $59(53.6)$ & $6(4.1)$ \\
\hline Yes, always & $51(46.4)$ & $97(66.4)$ \\
\hline \multicolumn{3}{|l|}{ Monthly income (Kyats) } \\
\hline Median (IQR) & $200,000(166,000)$ & $200,000(187,500)$ \\
\hline Range & $15,000-900,000$ & $15,000-2,000,000$ \\
\hline
\end{tabular}

All MSM in the study have experienced sexual intercourse with men in their lifetime. Mean age of first sex among them was $17.3 \pm$ 3.1 and ranged from 11 to 27 years. The proportions of MSM who had sex with men were around 67\% in both groups within one month. In addition, about $75 \%$ of young MSM and $87.7 \%$ of adult MSM had sex within six months. Similarly, 84.5\% of young MSM and $91.8 \%$ of adult MSM had sex within one year (data not shown in table). Table (2) shows the number of sexual partners in different time period according to age group of MSM. Having multiple sexual partners was seen in both age groups at all time periods. Within one month, $47.3 \%$ of young MSM and 63.9\% of adult MSM had multiple sexual partners. Over $70 \%$ to $80 \%$ of MSM had multiple sexual partners within six months and one year respectively. Similarly, $85.5 \%$ and $96.6 \%$ of MSM had multiple sexual partners in their life time.

Table (2) Number of sexual partners in different time period according to age group of MSM

\begin{tabular}{|c|c|c|c|}
\hline & \multicolumn{2}{|c|}{ Age group } & \multirow[b]{2}{*}{ p-value } \\
\hline & $\begin{array}{c}\text { 18-24 years } \\
\text { n(\%) }\end{array}$ & $\begin{array}{c}\geq 25 \text { years } \\
n(\%)\end{array}$ & \\
\hline Multiple partners within one month & $(\mathrm{n}=74)$ & $(\mathrm{n}=97)$ & $<0.05$ \\
\hline No & $39(52.7)$ & $35(36.1)$ & \\
\hline Yes & $35(47.3)$ & $62(63.9)$ & \\
\hline Multiple partners within six months & $(\mathrm{n}=82)$ & $(n=128)$ & $>0.05$ \\
\hline No & $24(29.3)$ & $35(27.3)$ & \\
\hline Yes & $58(70.7)$ & $93(72.7)$ & \\
\hline Multiple partners within one year & $(\mathrm{n}=93)$ & $(\mathrm{n}=134)$ & $>0.08$ \\
\hline No & $24(25.8)$ & $22(16.4)$ & \\
\hline Yes & $69(74.2)$ & $112(83.6)$ & \\
\hline Multiple partners within life time & $(\mathrm{n}=110)$ & $(n=146)$ & $<0.01$ \\
\hline No & $16(14.5)$ & $5(3.4)$ & \\
\hline Yes & $94(85.5)$ & $141(96.6)$ & \\
\hline
\end{tabular}

Condom use with permanent and non-permanent sexual partners according to age group of MSM is shown in Table (3). Never/inconsistent condom use was detected among 61.3\% of young MSM and 56.1\% of adult MSM who had sex with their permanent sexual partners within one month. On the other hand, 33.3\% of young MSM and 23.5\% of adult MSM did not use condom regularly during one month. 
Table (3) Condom use with permanent partners and non-permanent partners according to age group of MSM

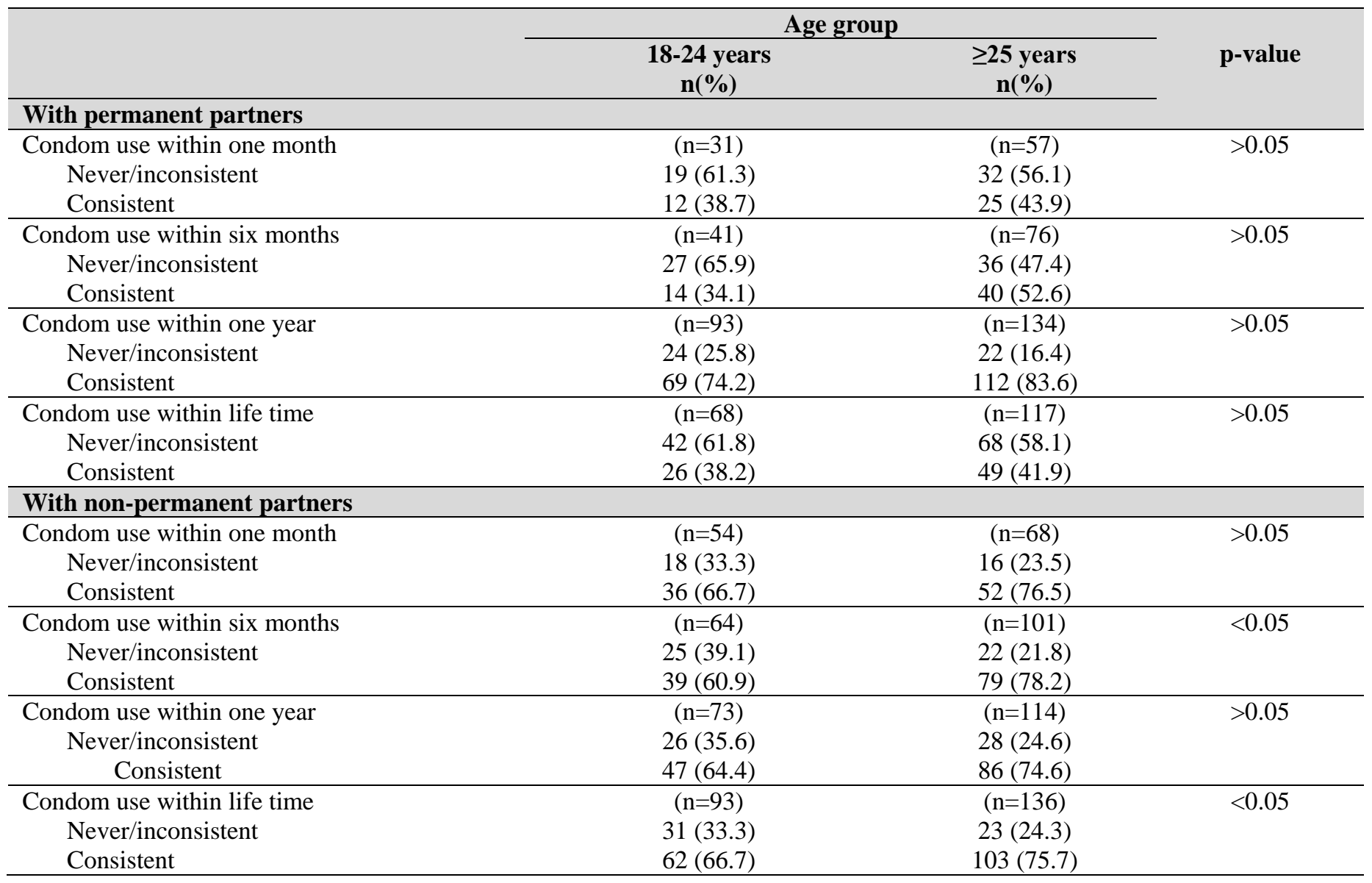

Number of sexual partners of MSM within different time periods according to the type of MSM is described in Table (4). Association was detected between type of MSM and number of sexual partners in one month. Significantly higher proportion of Apwint (open type) of MSM had more than one sexual partner than other types of MSM ( $<<0.001$ ). Over 69\% of Apwint (open type) of MSM, $51.3 \%$ of Apone (hidden type) and $26.5 \%$ of Thange (partner of MSM) had more than one sexual partner. Similar pattern of association between type of MSM and number of partners was also seen for other time periods like within three months, six months and one year $(\mathrm{p}<0.001)$.

Table (4) Number of sexual partners of MSM at different time periods according to the type of MSM

\begin{tabular}{|c|c|c|c|}
\hline & \multicolumn{2}{|c|}{$\begin{array}{l}\text { No. of sexual partners within } 1 \text { month } \\
\qquad \mathrm{n}=171, \mathrm{n}(\%)\end{array}$} & \multirow[t]{2}{*}{$\mathrm{p}$ value } \\
\hline & One partner & $>$ one partner & \\
\hline Type of MSM & & & $<0.001$ \\
\hline Apwint (Open type) & $30(30.6)$ & $68(69.4)$ & \\
\hline Apone (Hidden type) & $19(48.7)$ & $20(51.3)$ & \\
\hline \multirow{3}{*}{ Thange (Partner of MSM) } & $25(73.5)$ & $9(26.5)$ & \\
\hline & \multicolumn{2}{|c|}{$\begin{array}{l}\text { No. of sexual partners within } 6 \text { months } \\
n=210, n(\%)\end{array}$} & $\mathrm{p}$ value \\
\hline & One partner & $>$ one partner & \\
\hline $\begin{array}{l}\text { Type of MSM } \\
\text { Apwint (Open type) } \\
\text { Anone (Hidden tyne) }\end{array}$ & $\begin{array}{l}18(16.2) \\
20(36.4)\end{array}$ & $93(83.8)$ & $<0.001$ \\
\hline
\end{tabular}




\begin{tabular}{|c|c|c|c|}
\hline Thange (Partner of MSM) & $21(47.7)$ & $23(52.3)$ & \\
\hline & \multicolumn{2}{|c|}{$\begin{array}{l}\text { No. of sexual partners within one year } \\
n=227, n(\%)\end{array}$} & $\mathrm{p}$ value \\
\hline & One partner & $>$ one partner & \\
\hline Type of MSM & & & 0.001 \\
\hline Apwint (Open type) & $14(11.5)$ & $108(88.5)$ & \\
\hline Apone (Hidden type) & $17(27.0)$ & $46(73.0)$ & \\
\hline \multirow[t]{3}{*}{ Thange (Partner of MSM) } & $15(35.7)$ & $27(64.3)$ & \\
\hline & \multicolumn{2}{|c|}{$\begin{array}{l}\text { No. of sexual partners within lifetime } \\
\qquad \mathrm{n}=, \mathrm{n}(\%)\end{array}$} & $\mathrm{p}$ value \\
\hline & One partner & $>$ one partner & \\
\hline Type of MSM & & & $<0.001$ \\
\hline Apwint (Open type) & $3(2.3)$ & $126(97.7)$ & \\
\hline Apone (Hidden type) & $3(4.4)$ & $65(95.6)$ & \\
\hline Thange (Partner of MSM) & $15(25.4)$ & $44(74.6)$ & \\
\hline
\end{tabular}

\section{DisCUSSIONS}

The current study identified the sexual risk behaviors among MSM in major cities of Myanmar. Being consistent with IBBS data in 2015, mean age at first anal sex was 17 years in present study. Moreover, proportions of MSM who had sex within one year were around $85 \%$ in young MSM and $90 \%$ in adult MSM, significantly higher than that was reported in IBBS (14\% to 20\%) (National_AIDS_Program).

Sexual risk behaviors like having multiple sexual partners and having condomless anal sex were prevalent among MSM all over the world. The present study reported that nearly half of young MSM and over $60 \%$ of adult MSM had multiple sexual partners within one month, nearly three-fourth within six months and over three-fourth within a year. Increasing proportions of MSM were having multiple partners in different time periods.

The present study highlighted that consistent condom use among MSM depended on the type of sexual partners in which it was higher with non-permanent sexual partners than with permanent partners. Around one fourth to one third of young MSM never or inconsistently used condom with permanent sexual partners at different time periods like one month, three months, six months, one year and lifetime. Similarly, nearly $16 \%$ to $60 \%$ of adult MSM never or inconsistently used condom with non-permanent sexual partners at different time periods.

Previous studies in other countries have also reported the presence of multiple partners and inconsistent condom use among MSM. In China, over $40 \%$ of MSM did not use condom within previous six months (Li et al.) whereas in Malaysia, about $60 \%$ had unprotected sex within six months (Koh et al.). Similarly, in US, 58\% of MSM reported condomless anal intercourse within six months (Santos et al.). Mean number of sexual partners within six months was reported as 11 in Malaysia study while it was stated as 22 in current study. In addition, over $70 \%$ of MSM in current study had multiple sexual partners within six months. Similar findings were seen in other countries in which about $60 \%$ of MSM had multiple partners in China, about $70 \%$ had multiple partners in Vietnam (Garcia et al.) and over $90 \%$ of MSM had multiple partners in US within six months (Santos et al.). On the other hand, university students had lesser partners than MSM whereas about one-third of university students had multiple partners within one year (Choudhry et al.).

Having multiple sexual partners was very common among MSM and it may lead the risk of sexually transmitted infections. According to the past studies, over half to two-third of MSM had multiple sexual partners and had six to ten sexual partners within previous year. One of the studies stated that, there was sexual relationship with ten sexual partners within last one year (Chittamuru et al.). According to a systematic review of studies done in high income countries documented that there was an increasing trend of condomless anal 
intercourse among MSM but a decreasing trend in number of partners (Hess et al.). Possible explanations for the decreasing trend of condom use included availability of other HIV prevention options and optimism about HIV treatments.

In conclusion, intervention to reduce unsafe sexual practices like having multiple partners and condomless anal intercourse should be carried out since many MSM in the current study had practiced these unsafe sexual behavior.

\section{REFERENCES}

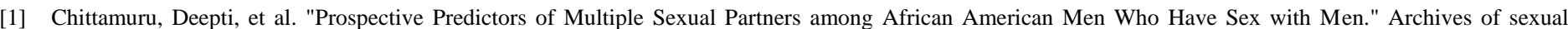
behavior 47.7 (2018): 2081-90. Print.

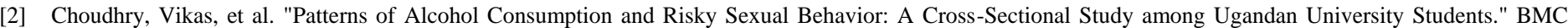
public health 14.1 (2014): 128. Print.

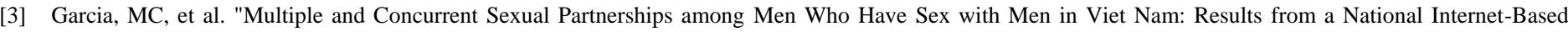
Cross-Sectional Survey." Health promotion international 31.1 (2016): 133-43. Print.

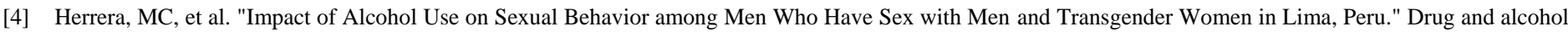
dependence 161 (2016): 147-54. Print.

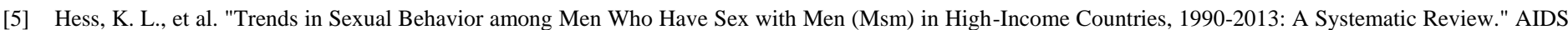
Behav 21.10 (2017): 2811-34. Print.

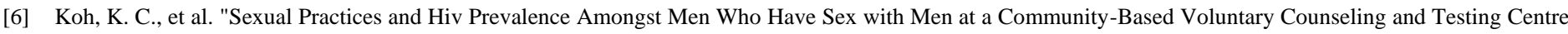
in Malaysia." ISRN Infectious Diseases 2013 (2013): 1-6. Print.

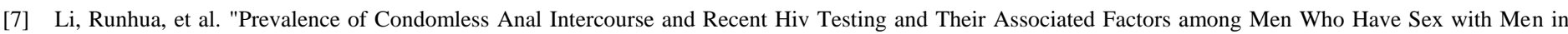
Hangzhou, China: A Respondent-Driven Sampling Survey." PloS one 12.3 (2017): e0167730. Print.

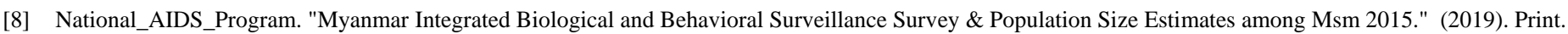

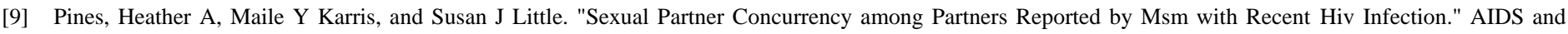
Behavior 21.10 (2017): 3026-34. Print.

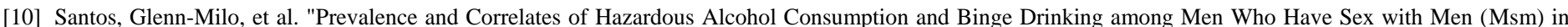
San Francisco." PloS one 13.8 (2018): e0202170. Print.

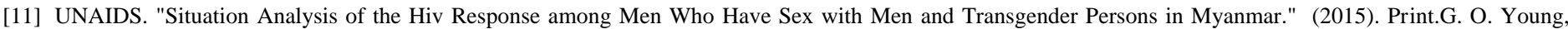

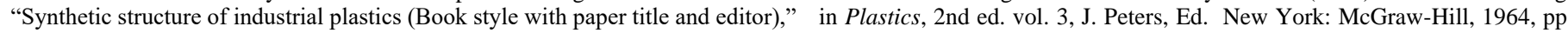
15-64.

\section{AUTHORS}

First Author - Kyaw Min Htut: Department of Medical Research, Ministry of Health and Sports, Yangon, Myanmar College of Public Health Sciences, Chulalongkorn University, Bangkok, Thailand 84kyawminhtut@gmail.com

Second Author - Chitlada Areesantichai, Drug Dependence Research Centre, WHO Collaborating Centre for Research and Training in Drug Dependence, Health and Social Science and Addition Research Unit, College of Public Health Sciences, Chulalongkorn University, Bangkok, Thailand

Third Author - Myo Myo Mon, Department of Medical Research, Ministry of Health and Sports, Yangon, Myanmar mmyomon@gmail.com

Fourth Author - Phyo Aung Naing, Department of Medical Research, Ministry of Health and Sports, Yangon, Myanmar phyoaungnaing84@gmail.com

Correspondence Author - Kyaw Min Htut: Department of Medical Research, Ministry of Health and Sports, Yangon, Myanmar College of Public Health Sciences, Chulalongkorn University, Bangkok, Thailand 84kyawminhtut@gmail.com 